\title{
Estimation of dislocation density from precession electron diffraction data using the Nye
}

Tensor

\author{
A.C. Leff ${ }^{1}$, C.R. Weinberger ${ }^{2}$, and M.L. Taheri ${ }^{{ }^{*}}$ \\ ${ }^{1}$ Department of Materials Science \& Engineering, Drexel University, Philadelphia, PA \\ ${ }^{2}$ Department of Mechanical Engineering and Mechanics, Drexel University, Philadelphia, PA \\ *Author to whom correspondence should be addressed: mtaheri@coe.drexel.edu
}

Keywords: Nye tensor, automated crystallographic orientation mapping in transmission electron microscopy (ACOM-TEM), dislocation density, plastic deformation

\begin{abstract}
The Nye tensor offers a means to estimate the geometrically necessary dislocation density of a crystalline sample based on measurements of the orientation changes within individual crystal grains. In this paper, the Nye tensor theory is applied to precession electron diffraction automated crystallographic orientation mapping (PED-ACOM) data acquired using a transmission electron microscope (TEM). The resulting dislocation density values are mapped in order to visualize the dislocation structures present in a quantitative manner. These density maps are compared with other related methods of approximating local strain dependencies in dislocation-based microstructural transitions from orientation data. The effect of acquisition parameters on density measurements is examined. By decreasing the step size and spot size during data acquisition, an increasing fraction of the dislocation content becomes accessible. Finally, the method described herein is applied to the measurement of dislocation emission during in situ annealing of $\mathrm{Cu}$ in TEM in order to demonstrate the utility of the technique for characterizing microstructural dynamics.
\end{abstract}

\section{Introduction}

Dislocations in the lattice of crystalline materials alter their mechanical and electrical properties and play a vital role in microstructural evolution [1][2][3][4][5]. Processes of microstructural evolution such as recovery and recrystallization rely on the stored energy from dislocations to drive them [1][2][6]. These relationships can be exploited through controlled thermomechanical processing in order to produce microstructures that optimize desirable properties such as ductility or corrosion resistance. Therefore, the ability to quantitatively measure dislocation densities is crucial to the understanding of deformation, recovery, and recrystallization, and the specific mechanisms by which these processes are driven. The unambiguous quantification of dislocation densities is difficult, however, and current methods based on electron backscatter detection (EBSD) methods can be limited by their spatial resolution and are only applicable to samples that can be analyzed in an SEM. To complement existing techniques and to develop higher resolution methods with higher dislocation density resolutions using automated processes, 
we propose a new method to quantify local dislocation densities. This method uses precession electron diffraction (PED) [7][8][9][10] data acquired in a transmission electron microscope (TEM) to generate quantitative dislocation density values based on the Nye tensor dislocation theory [11] for which spatial resolution is limited only by the TEM beam size. It relies on small shifts in orientation rather than relative strains, so that the strain tensor need not be accessible in order to quantify dislocation density.

42 The prevailing method for automated dislocation density measurements relies on the analysis of EBSD patterns in conjunction with Nye's dislocation density tensor to estimate dislocation densities. Nye's dislocation density tensor [11] relates the dislocation density at a point to the Burgers vector and the dislocation line direction as:

$$
\alpha_{i j}=n b_{i} r_{j}
$$

Where $\mathbf{r}$ and $\mathbf{b}$ are the unit and Burgers vectors of the dislocation $\mathrm{s}$, and $n$ is the number of dislocations intersecting with a unit area normal to $\mathbf{r}$. The dislocation density tensor defined this way only represents the net dislocation density for the given volume over which it applies and is related to the curvature of the material at that point. Thus, Nye's dislocation density tensor represents the geometrically necessary dislocations (GNDs) for a given volume or material point. The total dislocation density is a combination of these geometrically necessary dislocations as well as those that don't relate to curvature and thus don't have a net Burgers vector, which are termed statistically stored dislocations (SSDs). Arsenlis and Parks [12] pointed out, however, that the previous statement is only true if the SSD structures are fully contained within the measured volume. As the volume over which a given material is probed is decreased toward the length scale of the dislocation spacing, however, information regarding all dislocations should be accessible [13]. This concept is illustrated in Error! Reference source not found.. If smaller subdivisions are used, however, the dislocations are seen to contribute locally, even though the alternating curvatures they induce cancel one another out over a larger length scale.

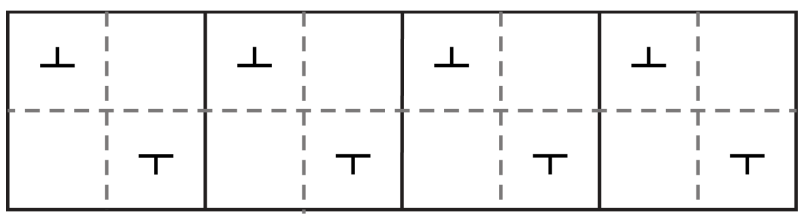

Figure 1. Schematic showing statistically stored dislocation structures inside of a region subdivided into even areas of two sizes (solid and dashed lines). The dislocations shown have opposite Burgers vectors so they will not contribute to lattice curvature from point to point if the large area sections are considered.

As the volume analyzed shrinks to zero, the measured dislocation density tensor approaches the true dislocation density, which is approximated as the L1 norm of the dislocation density tensor normalized by the burgers vector, as shown in equation 2 [14][13]: 
70 Therefore, spatial resolution plays a key role in accessing all of the information regarding

71 dislocation densities, both statistically stored and geometrically necessary.

72 One drawback to the estimation of dislocation densities using this method is that it ignores

73 curvature that results from residual elastic strain. The development of automated acquisition and

74 analysis of electron backscatter diffraction (EBSD) patterns in scanning electron microscopes

75 (SEMs) [15] has allowed for the application of the Nye tensor model to experimental results

76 [16][17][18], and has allowed for analysis of elastic strain. Kysar, et al. [16][17] demonstrated

77 that with a sample in a plane strain condition it is possible to estimate the GND density for

78 individual slip systems as well as the lower-bound GND density using variations of equations 1

79 and 2 using the point to point changes in orientation determined using EBSD. The cross-

80 correlation technique developed by Wilkinson, et al. [19] for the analysis of high resolution

81 EBSD patterns has allowed for the precise quantification of the elastic strain and rotation tensors.

82 Several groups have taken advantage of this newly accessible information to calculate the Nye

83 tensor using Kroner's analysis [13][20][21][22][23][24]. In doing so, however, Wilkinson, et al.

84 determined that the contribution of elastic strain is insignificant compared to lattice rotation in

85 contributing to overall lattice curvature of plastically deformed metals [20], justifying the

86 application of Nye's original treatment without the addition of terms to incorporate elastic strain

87 [21][22]. Likewise, recent work by Ruggles and Fullwood shows that for data acquired using

88 EBSD incorporation of elastic strain measurements in addition to rotation does not significantly

89 increase the accuracy of the calculation [13].

90 Other techniques exist for mapping elastic and plastic strain using EBSD patterns, including

91 image quality analysis [25][26] and local misorientation mapping methods such as kernel

92 average misorientation (KAM) analysis, which was introduced by D.P. Field, et al. [27][26].

93 While these methods are less quantitative than the cross-correlation approach with respect to

94 dislocation density measurement, they are able to yield data that can be used to estimate the

95 relative dislocation densities. Another method for quantifying dislocation densities from EBSD is

96 the weighted Burgers vector analysis developed by Wheeler, et al. [28]. The weighted Burgers

97 vector is defined as the sum of the densities of each type of dislocation multiplied by their

98 Burgers vectors. This method has the advantage that there is no information about the $\mathrm{Z}$ axis

99 required to define it fully.

100 Despite recent improvements in resolution, conventional EBSD on bulk samples in SEMs is

101 limited to a minimum spatial resolution of approximately 20-40 nm depending on the material

102 being examined [29][22][30]. This drawback, along with its nature as a surface characterization

103 technique, makes SEM less than ideal for measuring dislocations. TEM is an obvious choice as a

104 replacement technique due to its superior resolution and because, being a transmission technique,

105 it is capable providing images of the dislocation structure to accompany the quantitative

106 evaluation using the Nye tensor. This allows for a visual comparison of the resulting GND 
107 density estimate with the dislocations present in the accompanying TEM micrograph and 108 provides useful input for scanning parameters, such as approximate dislocation spacing and 109 subgrain sizes. This point was made by Wilkinson, et al. [19], who touched on the possible 110 advantages of using diffraction patterns acquired in TEM when the cross-correlation EBSD 111 technique was first described. The one drawback of using a transmission technique is that the 112 sample has two free surfaces so more relaxation of the defect structure can occur. It is important 113 to note that advancements in transmission Kikuchi diffraction (TKD) have shown promise for 114 decreasing the spatial resolution of EBSD systems below $10 \mathrm{~nm}$ for electron-transparent thin 115 foils, making TKD another viable alternative [30]. Since the specimens for TKD must be 116 electron-transparent the sample preparation for this technique is similar to that of TEM. It is 117 critical to note, however, that while TKD provides advantages in angular resolution, it still lacks 118 the ability to directly image the internal defect structures simultaneously.

119 One approach to dislocation density measurement in TEM is the analysis of precession electron 120 diffraction (PED) patterns [7]. PED is diffraction acquired using a hollow cone of illumination 121 produced by rapidly precessing the beam in a circle [7]. The use of PED allows for quasi-

122 kinematical diffraction conditions that make visible the reflections from the higher order Laue 123 zones (HOLZ) that contain information about the structure parallel to the optic axis that is 124 typically inaccessible in spot diffraction patterns [31].

125 Automated crystallographic orientation mapping in TEM (ACOM-TEM) [8][9][10] provides a 126 potential source of input data for dislocation density calculations based on the Nye tensor; when 127 used in conjunction with PED, it provides three-dimensional orientation information as well as 128 the displacement in two dimensions. In this paper, we apply existing methods for quantifying 129 plastic deformation from orientation data and present a method for estimating and mapping the 130 dislocation density in TEM specimens using the Nye tensor utilizing Euler angle and 131 displacement input from ACOM-TEM [32]. By calculating the best fit of the tensor describing 132 the contortion of the lattice for each point with respect to its nearest neighbors, changes in the 133 dislocation density from point to point can be measured on a nanometer length-scale. A similar 134 methodology was first described by Pantleon [18] using conventional EBSD data as inputs. An 135 alternate approach to applying the Nye tensor to ACOM-TEM to quantify dislocation density 136 was recently demonstrated by Ghamarian, et al. [33]. That method, which was developed 137 concurrently with the one described herein, is similar in approach but the dislocation density 138 maps produced appear very different. The primary difference is the use of Kuwahara filtering to 139 manipulate the orientation data prior to calculating the Nye tensor, which is addressed in Section 140 4.4. Additionally, it is unclear from [33] how the Nye tensor values for each individual point are 141 averaged and assigned. This analysis technique is capable of accessing both GNDs and SSDs due 142 to the scale of the applied probe. It has been applied to the study of the effect of dislocation 143 densities on microevolution processes such as grain rotation, boundary migration, and other 144 microstructural transitions [34]. Maps produced using this technique are consistent with TEM 145 images of dislocation structures taken in the analysis regions, and the dislocation density values 
calculated are on the same orders of magnitude with those from literature [35][22][36]. The results generated by this method presented herein for quantification of both geometrically necessary and statistically stored dislocation content is compared to those generated by related methods for analyzing dislocation dependent microstructural events, such as kernel average misorientation and local orientation spread.

\section{Material and Methods}

\subsection{Sample Preparation}

Cold-rolled microcrystalline oxygen-free electronic copper ( $99.99 \%$ pure) half-inch plate (McMaster Carr) was cut into $1.25 \times 1.25 \mathrm{~cm}$ strips and annealed for one hour at $540^{\circ} \mathrm{C}$ to achieve a fully recrystallized microstructure. The strips were then subjected to various additional rolling reductions ranging from 5 to 50 percent in order to achieve a range of dislocation densities. Some specimens were subjected to partial annealing at temperatures ranging from 400$720{ }^{\circ} \mathrm{C}$ in order to produce microstructures in the various stages of recovery and recrystallization. TEM disc specimens were cut from the material perpendicular to the rolling direction and prepared using conventional metallographic techniques and jet polished using a Struers TenuPol-5 electropolisher. In addition, site-specific lift-outs of individual grain boundaries were prepared using an FEI Strata DB235 focused ion beam (FIB).

\subsection{Precession Diffraction Pattern Acquisition}

The NanoMEGAS SPINNING STAR ${ }^{\mathrm{TM}}$ precession electron diffraction and ASTAR ${ }^{\mathrm{TM}}$ ACOMTEM systems, integrated into a JEOL 2100 Lab6 TEM, were used to acquire the orientation data used as the input for GND density calculations [32]. A precession angle of $0.60^{\circ}$ was utilized for all experiments. Step sizes of $10.40 \mathrm{~nm}$ and $3.90 \mathrm{~nm}$ for both $\mathrm{x}$ and y directions were used. The beam spot size used was $10 \mathrm{~nm}$. The patterns were acquired and indexed automatically following a method first described by Rauch and Veron [8].

Because the step size and probe size for this setup are not linked there is a potential for error due to under- or over-sampling depending on the acquisition parameters. Likewise, the acquisition parameters ought to have a direct effect on the fraction of the dislocation that is geometrically significant at the length-scale being considered as previously discussed. A sampling model was produced to evaluate how these variables affect the values produced. As shown in Figure 2A, the model takes measurements of the average orientation across a width $\mathrm{D}$ that are a distance $\Delta$ apart. The specimen being measured contains an array of equally spaced dislocations $d$ apart that have either positive or negative signs assigned randomly. For a complete description of the 

detected $\left(\rho / \rho_{0}\right)$ increases as $\Delta$ is decreased. For all spot sizes tested the relationship between $\rho / \rho_{0}$ and $\Delta$ is the same until $\Delta / \mathrm{d}$ approaches one. For spot sizes approaching or decreasing beyond $\mathrm{d}$ this trend continues as $\rho / \rho_{0}$ approaches unity and levels off, meaning the total dislocation content is measured without over-sampling. No combination of $\Delta$ and $\mathrm{D}$ lead to an overestimation of dislocation density and the systematic error caused by under-sampling can be corrected for, especially with iterative measurements with varied acquisition parameters.

A)

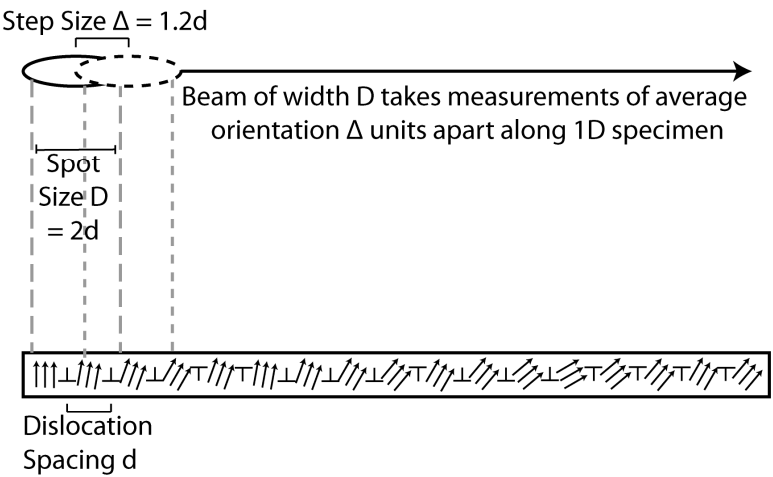

191

192

193

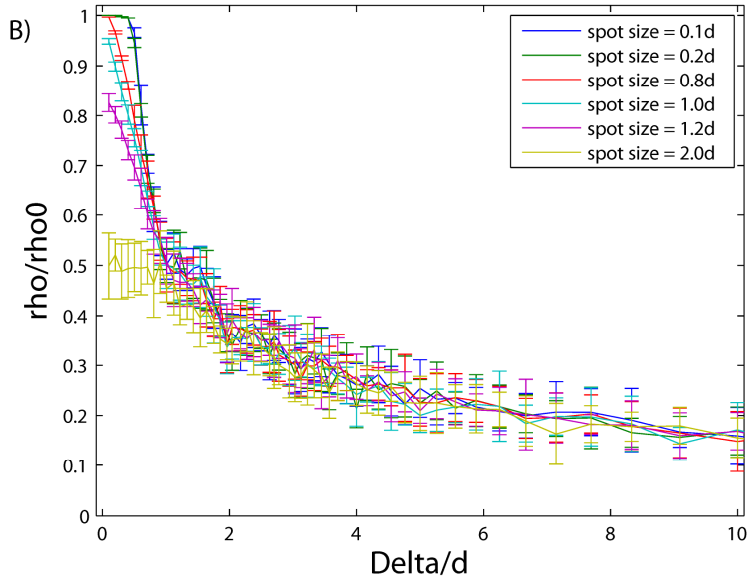

Figure 2. A) Schematic of sampling model. B) Fraction of dislocation density measured by simulation versus step size for various spot sizes. Each line is the average of 10 iterations with randomized dislocation signs.

\subsection{Local Orientation Spread and Dislocation Density Analysis}

The orientation data was analyzed using the TSL OIM Analysis software package developed by EDAX. Using this software, maps of local orientation spread and kernel average misorientation, metrics typically used to assess local deformation in SEM specimens analyzed using EBSD, were generated in order to qualitatively visualize internal strains due to lattice curvature. This serves as a rough approximation of GND density because any curvature within an individual grain must be accommodated by dislocations [26]. Local spread is calculated by treating each point in the scan as the center of a kernel of $n$ nearest neighbors then averaging the misorientation between each pair of points within the kernel and assigning the mean value to the center point. Kernel average misorientation is calculated similarly, but instead of considering each pair of points, only the misorientation between the center point and the other points are used. A kernel size of $n=3$ and exclusion angle of $5^{\circ}$ was chosen for all data for the purpose of consistency. Schematic representations of these calculations are shown in Figure 3 along with an example map for each. Although it has not previously been performed with crystal orientation data acquired in the TEM, this method has been used extensively for the analysis of EBSD data as discussed in Section 1 [27][26]. 
A)

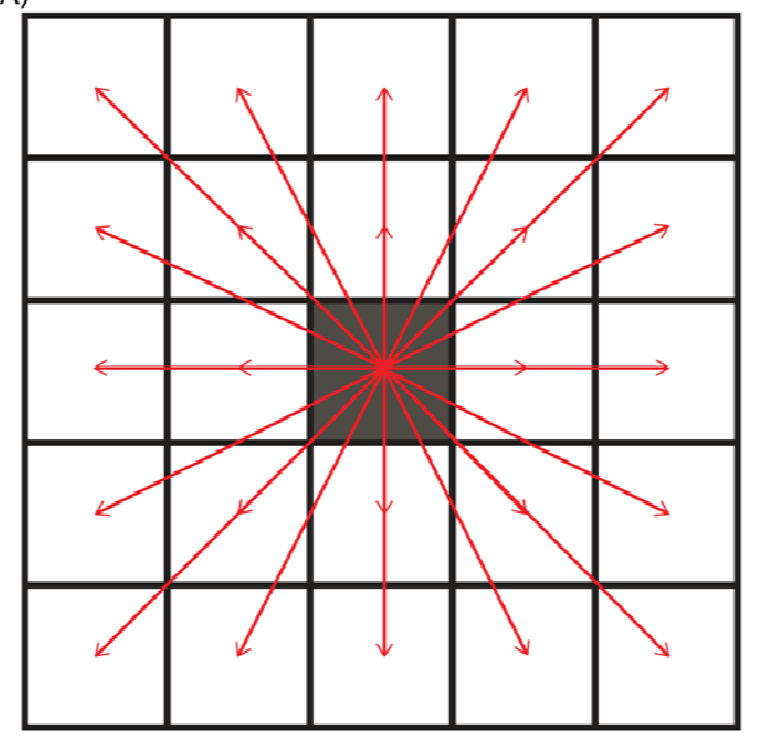

C)

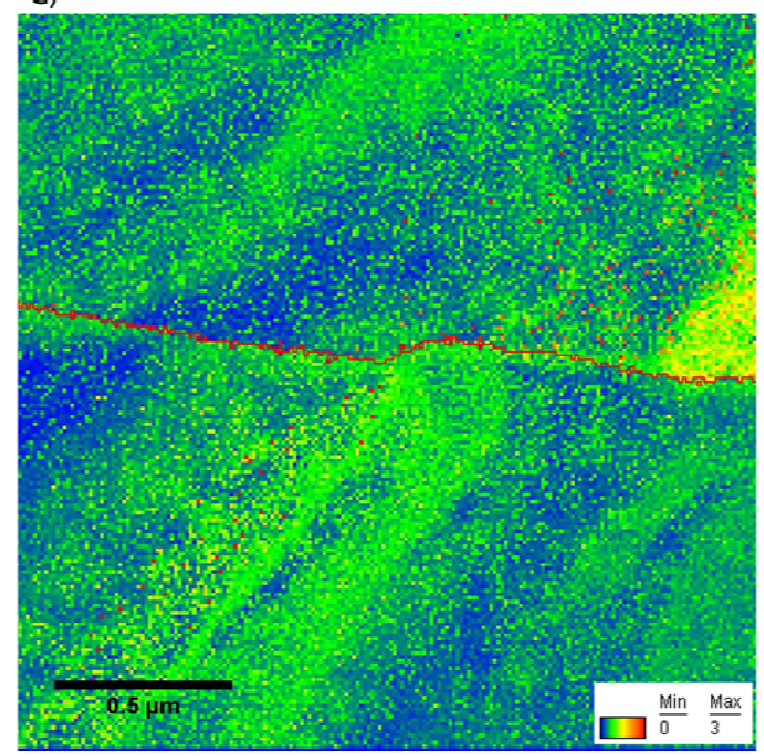

B)

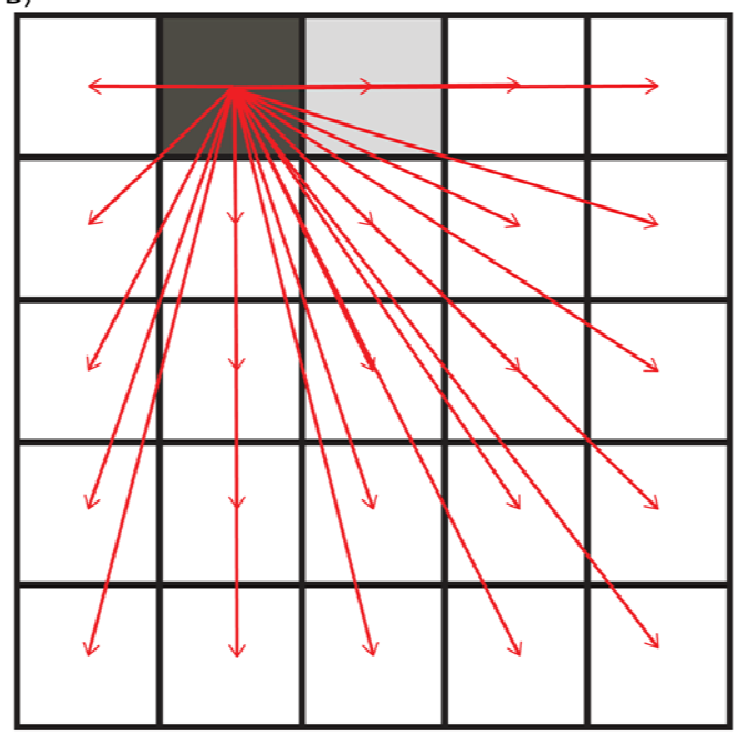

D)

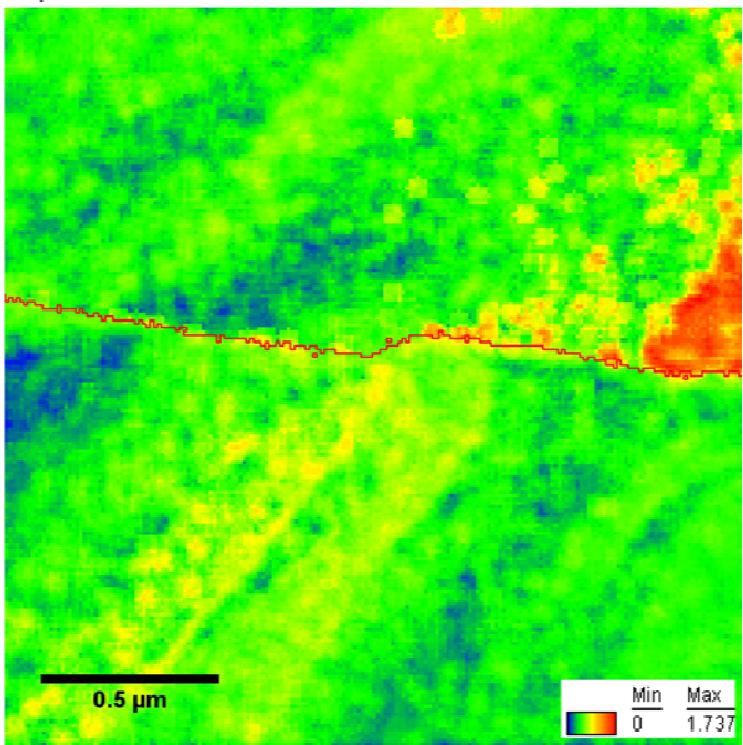

Figure 3. A\&B) Schematic representations of kernel average misorientation and local spread calculations, respectively. C\&D) Kernel average misorientation and local orientation spread maps of a region adjacent to a $\Sigma 3$ grain boundary in $\mathrm{Cu}$.

218 In order to obtain a more accurate, quantitative estimate of the GND density beyond local

219 orientation changes, the contortion tensors relating each point to its eight nearest neighbors were

220 calculated. Each point was considered as the center of a kernel with a radius of one and the 221 tensor components relating the eight neighbors in the kernel to it were determined. A least222 squares fitting was then applied to calculate a single contortion tensor that best describes the 223 relationship between the center point and its neighbors. These least-squares contortion values 224 were then used to calculate a single Nye tensor that describes the state of dislocation at each 225 point. While there exists a wide range of approaches to calculating a set of Nye tensor values to 
describe a given unit volume because each set of tensor values describes the dislocation content required to accommodate the disorientation between two known points, the method described herein avoids both directionality (i.e. comparing each point to its right-hand neighbor) and the use of a reference or average orientation. It is not clear that previous efforts [33] addressed this critical point. Once the Nye tensor describing each point has been calculated, equation 2 is used to estimate the lower-bound GND density based on the assumption that all dislocations have a $1 / 2<110>$ Burgers vector [22][13] since $\mathrm{Cu}$ is FCC. For other crystal structures where only a single family of Burgers vectors is present (such as $1 / 2<111$ in BCC), this assumption is easily adapted. For crystal structures with more than one family of dislocations, such as HCP, a weighted average Burgers vector must be calculated [13]. Only five of the nine tensor components were directly accessible from the data as discussed in detail in Section 3 so only those components were taken into consideration. Contour plots of the estimated lower-bound GND density were generated in Origin ${ }^{\mathrm{TM}}$ for direct comparison with TEM micrographs of the regions analyzed.

\section{Nye Tensor Calculation}

243 follows:

Where $K_{k k}$ is in the Einstein summation notation implying that $K_{k k}=\sum_{i} K_{i i} . \delta_{\mathrm{ij}}=1$ for $\mathrm{i}=\mathrm{j}$ and $\delta_{\mathrm{ij}}=0$ for $\mathrm{i} \neq \mathrm{j}$. Nye defined the contortion as a second-rank tensor:

$$
\alpha_{i j}=K_{j i}-\delta_{i j} K_{k k}
$$

$\partial \varphi_{i}$ is the three-dimensional rotation vector and $\partial x_{j}$ is the displacement vector. These are generated using ACOM-TEM. First, the axis-angle pair for the disorientation between each point and its neighbors is calculated. The rotation vector $\partial \varphi_{i}$ is then approximated as the product of the axis and angle according to infinitesimal rotation theory [37]. $\partial x_{j}$ is the displacement between the steps being compared. Equation 6 is undefined whenever $j=3$ because the displacement along the z-axis is zero. These undefined values for $K_{i 3}$ will carry through when $K$ is plugged into equation 5, which is written explicitly as:

$$
\alpha_{i j}=\left(\begin{array}{ccc}
-K_{22}-K_{33} & K_{21} & K_{31} \\
K_{12} & -K_{11}-K_{33} & K_{32} \\
\underline{K}_{13} & \underline{K}_{23} & -K_{11}-K_{22}
\end{array}\right) .
$$
From this expression it is clear that the components $\alpha_{11}, \alpha_{22}, \alpha_{31}$, and $\alpha_{32}$ will be undefined with 


\section{Results and Discussion}

261 4.1 Local Orientation Spread

262 Maps of local orientation spread (LOS) were produced as a preliminary method for qualitatively

263 assessing strain using ACOM-TEM data [32] and to compare with studies performed by Field et

264 al. [38][26]. This technique provides a rough estimate of the strain in the lattice due to local

265 changes in the crystal orientation caused by the presence of dislocations or long-range elastic

266 strain gradients. A LOS map is shown in Figure 4 along with a corresponding TEM micrograph

267 and orientation map. The LOS maps are normalized to the minimum and maximum orientation

268 spread values present within the dataset, in which high angle grain boundaries (HAGBs) are

269 shown in black, $\Sigma 3$ boundaries in red, and low angle grain boundaries (LAGBs, $2-15^{\circ}$ ) in teal.

270 At high magnification, the width of the boundary is able to be resolved, and thus the orientation

271 is viewed clearly as a gradient across a finite distance (as opposed to an abrupt jump from point

272 to point in a scan line). As a result, the TSL software fails to identify the $7^{\circ}$ LAGB indicated

273 with the solid arrow in Figure 4 as a grain boundary, instead showing it as a wide orientation 

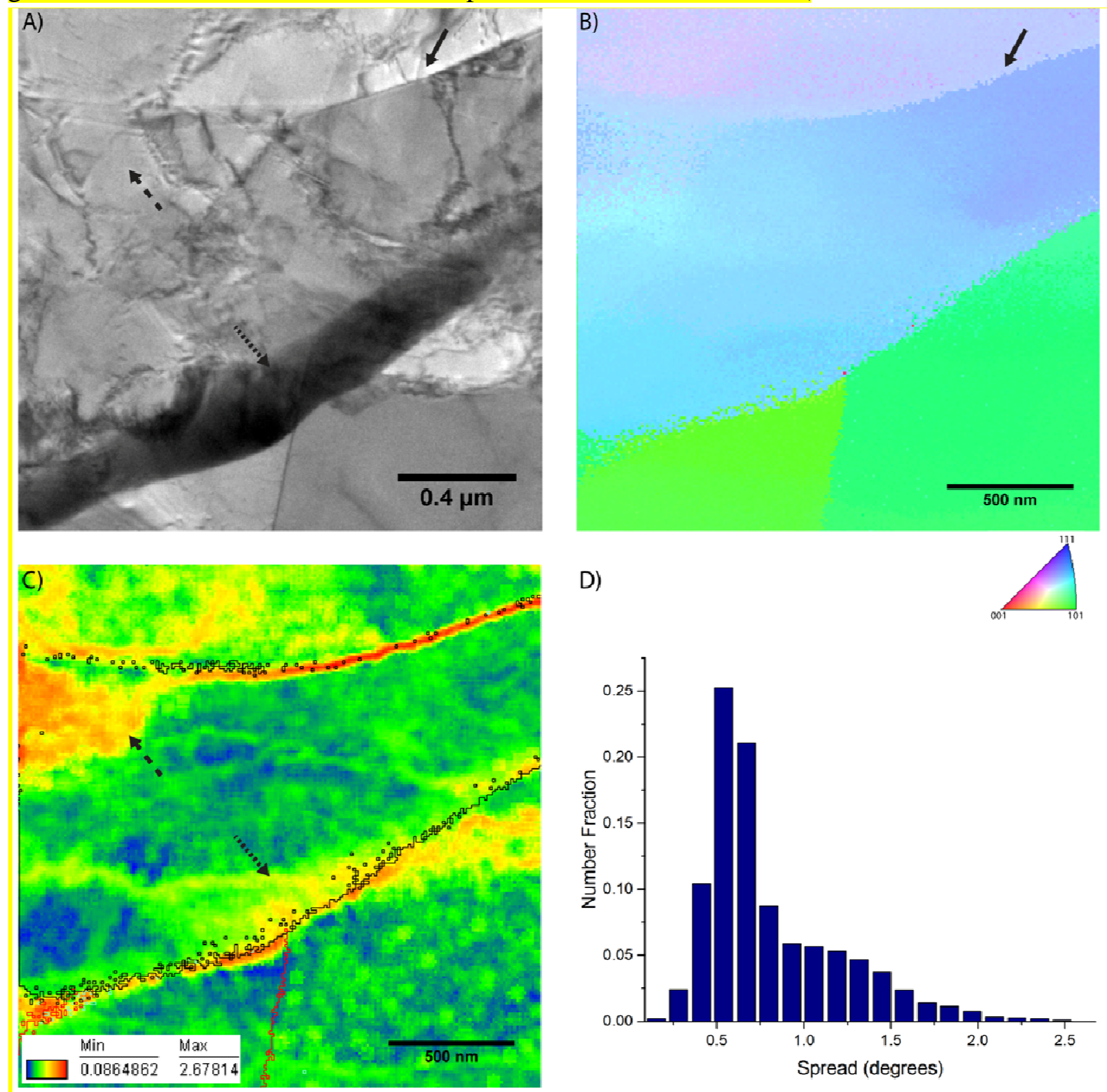

276 Figure 4D), which typically follows a log-normal distribution when LAGBs are not present, or at 277 spatial resolutions where their width is not resolved like in EBSD. It is important to note that 278 while some of the strain concentrations highlighted in the LOS map (Figure 4C), such as the one 279 indicated with the dotted arrow are readily visible in the corresponding TEM micrograph (Figure $2804 \mathrm{~A}$ ), others, such as the feature indicated with the dashed arrow, are not resolved. In addition, the 281 effects of bend contours are not present in the LOS maps, thus reducing the level of noise in the 282 image and facilitating dislocation analysis. The ability of this analysis to reveal dislocation 283 structures more readily than by TEM imaging alone demonstrates the importance of developing 284 characterization techniques capable of measuring dislocation content that can be utilized in a 285 complimentary fashion with TEM. 

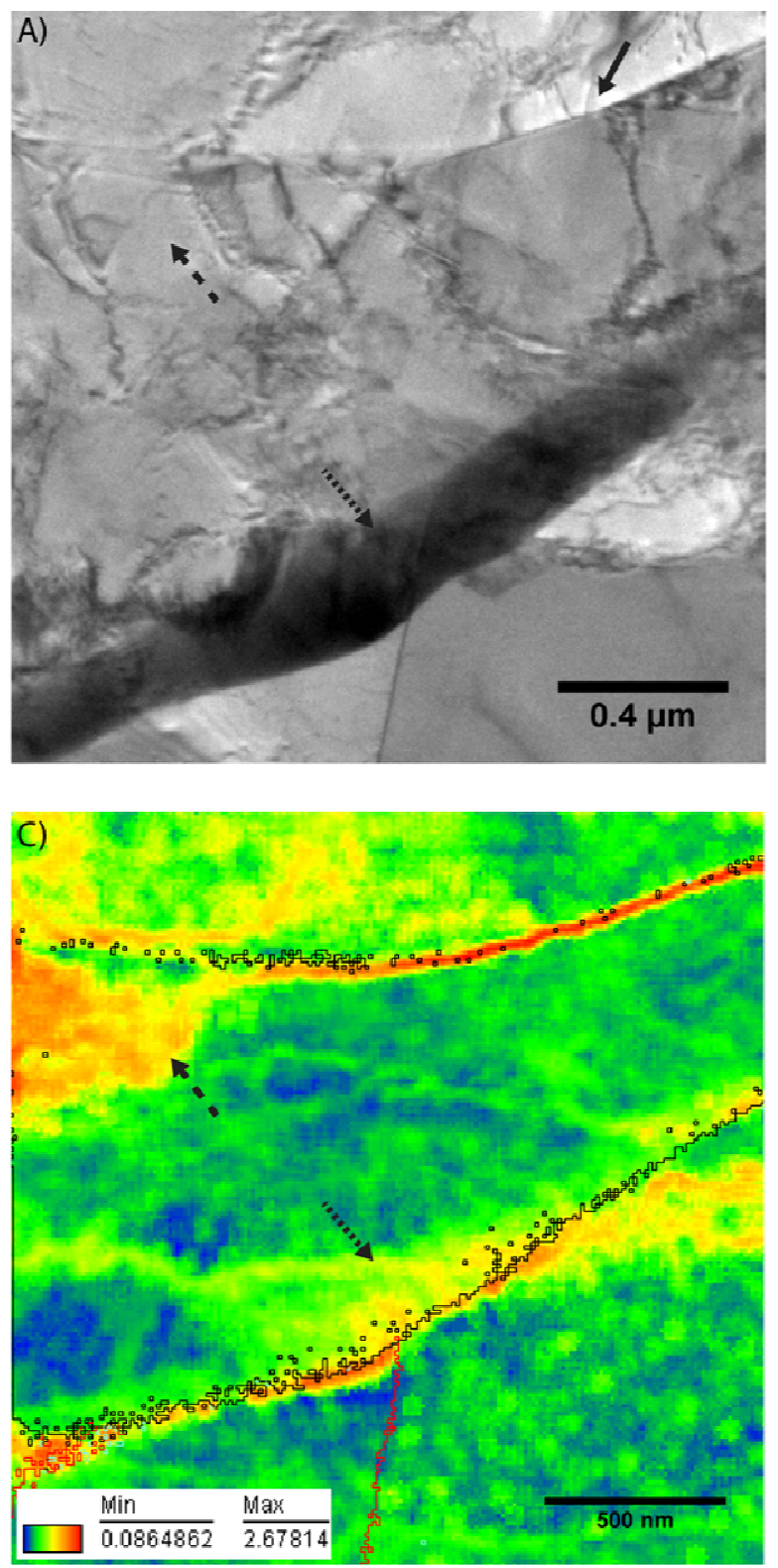
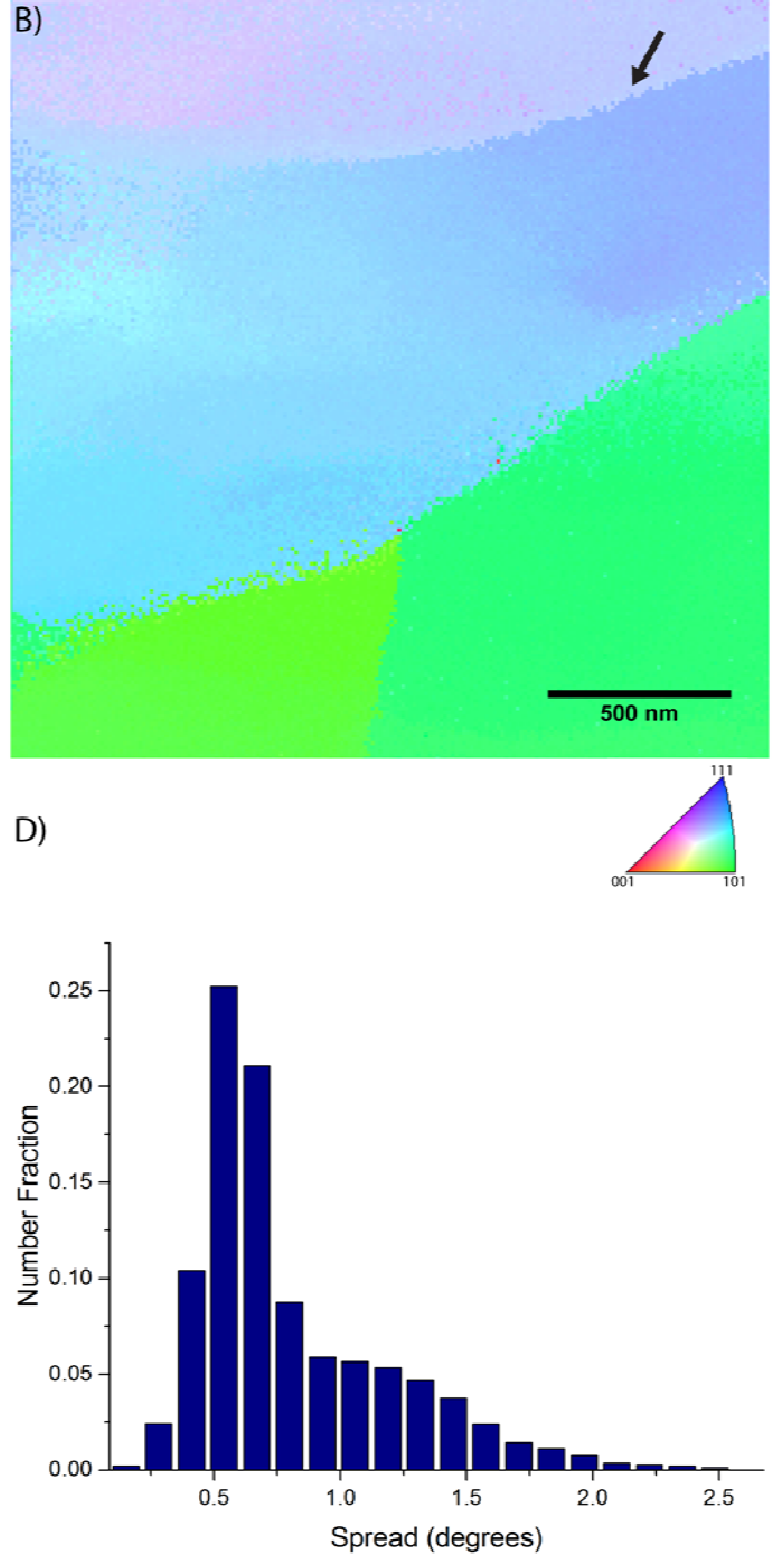

Figure 4. A) TEM micrograph of triple junction and LAGB. B) Corresponding inverse pole 290 figure map. C) Local orientation spread (3n). D) Number fraction distribution of local orientation 291 spread. 
Lower bound GND density (LB-GNDD) maps generated by the method described herein yield a quantitative view of dislocation structures. They show advantages over LOS maps in that the values correspond directly to the defects present in the specimen whereas the local spread only provides a relative comparison. Additionally, not all features of the dislocation structure are resolved by the LOS maps. Figure 5 shows a partially recovered region near a HAGB with corresponding LOS and LB-GNDD maps. The two maps are qualitatively similar, with small differences arising from the different number of nearest neighbors and maximum angles included in the calculations, but the GND density map provides a better quantitative representation of the defect distribution.

The GND density is plotted on a log scale in order to show the full range of values, which are high compared to those reported in the literature from EBSD data [35][22] $\left(10^{13}-10^{16} \mathrm{~m}^{-2}\right)$. This discrepancy can be attributed to the very fine step size used relative to EBSD measurements as well as differences in probe size and angular resolution [22], as is addressed in detail in the following section. Another factor that could contribute to the high density values is that all of the regions being examined are adjacent to grain boundaries. GND mapping in EBSD typically shows the highest densities in the regions along the boundaries, with low densities in the grain interiors. Another factor that contributes to the high GND density values reported herein is the minimum angular resolution of the indexing software used to determine the orientation at each point. The minimum resolution for the current indexing method with the current input parameters is approximately $0.5^{\circ}$. This is determined by the angular spacing between template patterns used for indexing. Any rotation that is smaller than this value but still detected by the software will be inflated to this minimum, artificially increasing the densities reported. Improvements to the angular resolution of pattern indexing will reduce the significance of this effect. A method was described recently to use the intensity of the spots to interpolate between template orientations in order to optimize the index quality [39]. By applying this orientation refinement algorithm, a resolution better than $0.3^{\circ}$ was reported. Application of this method in conjunction with the present analysis method is discussed in Section 4.4. Nonetheless, angular resolution is currently the primary drawback of using PED-ACOM data for strain mapping [33] and the main advantage of TKD as an orientation mapping technique for thin specimens [30]. One potential path forward for dislocation mapping in TEM at high angular resolution is the use of automated CBED [40].

324 The step size used for the acquisition of orientation data is more than an order of magnitude beneath the minimum dislocation cell size for copper of $\sim 250 \mathrm{~nm}$ as observed in literature[41][42]. As a result, this technique is able to resolve the walls and interiors of dislocation cells, even in metals that have undergone severe plastic deformation, as shown in Figure 5C\&E. It should be noted that the step sizes used in the analysis shown in Figure 5C were on the same order of magnitude as the dislocation spacing. As such, the measured volumes include fractions of defect structures that, taken together, would be considered as SSDs. As discussed in detail in Section I, if the complete SSD structure is not contained within the volume used for the calculation of the Nye tensor, then those dislocations will be accessible using this 
333 method. As a result, although the values reported herein are referred to as GNDs, they ought to 334 represent a larger fraction of the total dislocation density than GND densities calculated using 335 larger probe volumes. In Figure 5C the dislocation cell walls are clearly outlined, as expected, 336 but variations in dislocation density within the interiors of the individual cells due to SSDs are 337 also resolved as can be seen clearly in the blown up region of the map shown in Figure 5E.

338 It is important to note that if a high GND density is calculated based on the misorientation where

339 it is clear from microscopy that none is present then the curvature in the lattice at that point must 340 be due to elastic strain. It is not always clear, however, whether dislocations are present in a 341 given region due to the effect of diffracting conditions in the TEM on the visibility of defect 342 structures. Elastic strain cannot be measured using the current approach, although it can be 343 extracted from an on-axis nanobeam diffraction pattern by cross-correlation with a reference 344 pattern from an unstrained region. If significant elastic strains are present they will also 345 contribute to the inflation of GND density values for the present methodology since they are not 346 taken into consideration in the calculation so all orientation changes are attributed to 347 dislocations.

348 The primary drawback of the present analysis is the unknown thickness of the specimen along 349 the z-axis, which is similar to the difficulties encountered with EBSD being a surface technique. 350 This results in only five of the components of the Nye tensor being directly accessible [18]. 

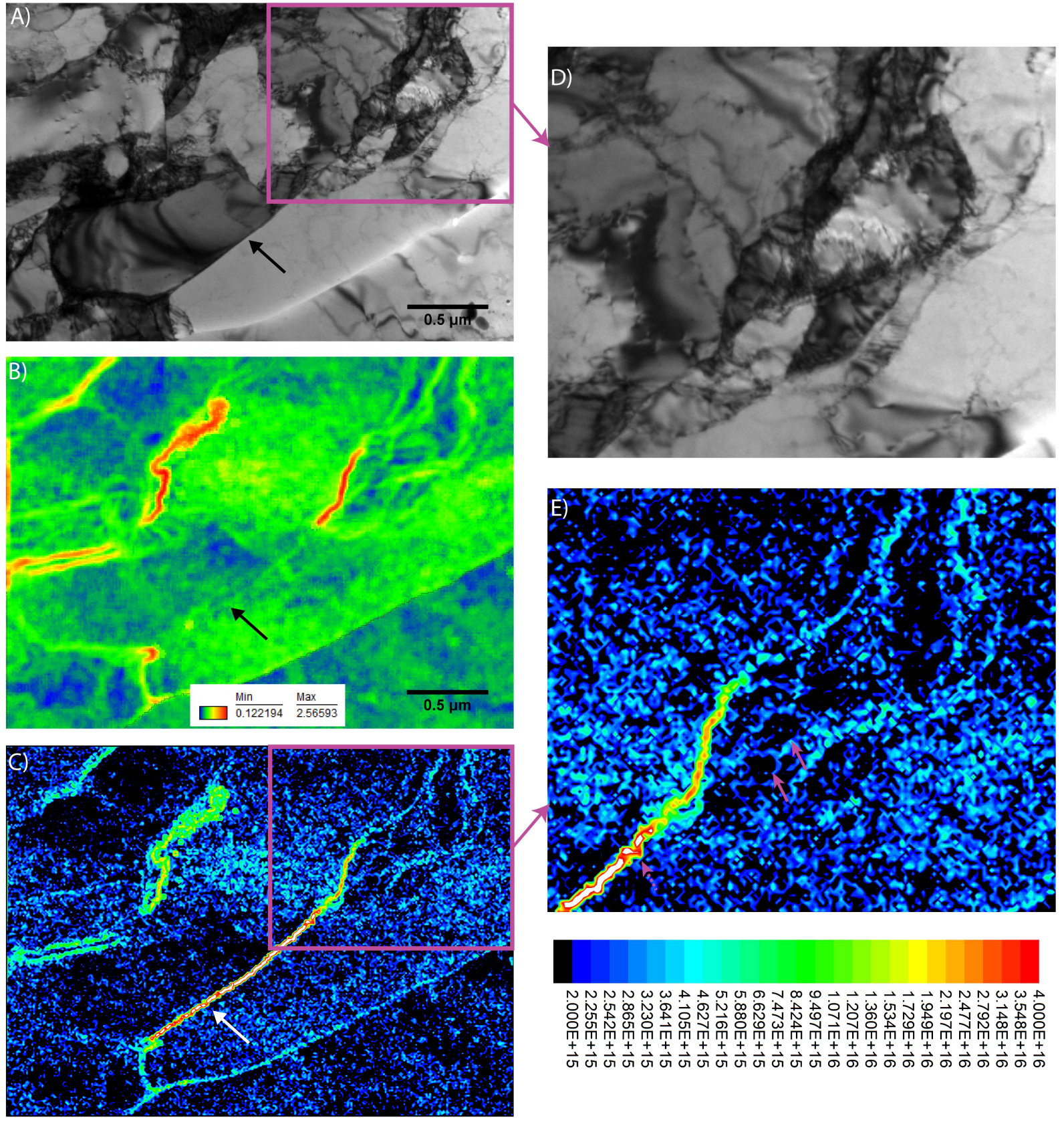

353 Figure 5. A) TEM micrograph of a HAGB in a region that has undergone a partial recovery 354 process. B) Local orientation spread map produced for the same region. C) Estimated lower355 bound GND density map. D) Expanded view of the region outlined in A. E) Expanded view of 356 region outlined in $\mathrm{C}$, which corresponds to D. Dashed arrow indicates LAGB. Solid arrows indicate dislocation cell walls. 
360 In order to assess the effect of step size used for data acquisition on the maps produced by this method, individual regions were scanned at a range of step sizes. Decreasing the step size independent of the probe size was shown to improve the spatial resolution of the technique. Most importantly, as predicted by the sampling model, the fraction of dislocations measured increases as $\Delta / \mathrm{d}$ is decreased. Jiang, et al. [22] observed the same phenomenon for data acquired using EBSD, noting that the density values calculated decrease when the data is binned to a lower spatial resolution.

An initial step size of $10.4 \mathrm{~nm}$ was chosen because it matches well with the minimum effective probe size of the TEM being used. Because the defect spacing and feature size is on the same order of magnitude, however, a smaller step size of $3.9 \mathrm{~nm}$ was used to analyze the effect of oversampling. As can be seen in Figure $6 \mathrm{E} \& \mathrm{~F}$, decreasing the step size results in an increased spatial resolution, as expected. The resulting maps reveal the GND structure in greater detail, removing much of the noise present in the maps produced with the larger step size. These same effects are clear from the LOS maps shown in Figure 6C\&D, where the qualitative details of the defect structure are much more visible at the smaller step size. The normalized dislocation density probability distribution for a given area remains approximately the same regardless of step size (Figure 6G and $\mathrm{H}$ ), following a log-normal relationship that is nearly identical with dislocation density probability distributions reported in the literature from EBSD-based analysis [43]. This indicates that a calibration factor can be developed in order to produce accurate, repeatable measurements of the dislocation density distribution regardless of the step size used. The average density for the first 780 by $780 \mathrm{~nm}$ area of each scan is $3.62 \times 10^{15} \mathrm{~m}^{-2}$ for the 10.4 $\mathrm{nm}$ step and $7.34 \times 10^{15} \mathrm{~m}^{-2}$ for the $3.9 \mathrm{~nm}$ step. These values were calculated by averaging the point densities across this area. This increase in measured dislocation density is due to the fact that an increasing fraction of dislocations are geometrically significant as the length-scale of the measurement is decreased due to short-range fluctuations in orientation produced even by dislocations traditionally considered to be SSDs as illustrated in Figure 1. This effect is especially drastic as step sizes decrease beyond the average dislocation spacing. A dislocation density of $7.34 \times 10^{15} \mathrm{~m}^{-2}$, the value produced using the $3.9 \mathrm{~nm}$ step, is equivalent to a mean dislocation spacing of $11.7 \mathrm{~nm}$, so the increase in measured dislocation density when the step size is decreased from 10.4 to $3.9 \mathrm{~nm}$ is expected according to the results of the sampling model shown in Figure 2B. The step size for acquisition can be further reduced, potentially down to a fraction of a nanometer. Doing so without also reducing the probe size of the TEM will result in further oversampling, with each pattern containing much the same information as the patterns acquired adjacent to it. Since this method relies on orientation measurements rather than a direct count, oversampling does not lead to over-counting, assuming that the orientation assigned is a weighted average of the distribution of orientations contained within the probe volume. If the measurement spacing is further reduced, eventually a critical step size $\left(\Delta_{\mathrm{c}}\right)$ will be reached where all dislocations are accessible. This $\Delta_{\mathrm{c}}$ is not a constant as it depends both on the minimum dislocation spacing present in the specimen and the probe size being used. Oversampling will, however, lead to increased density maxima and a higher number of points with low density 
400 values since the nominal area over which the density is calculated decreases but the probe area 401 stays the same. Since dislocations tend to be concentrated into tangles and cell walls, density 402 values from measurements taken in such regions will increase as step size is decreased because 403 the same number of dislocations will be probed over a smaller nominal area. The interiors of 404 dislocation cells will, however, contribute a higher fraction of data points as step size is

405 decreased. The interaction of these two effects is such that the average dislocation density for a 406 region should remain constant except for the effects of accessing SSDs as previously discussed. 407 One should note for experimental planning that decreasing the step increases acquisition time 408 scans. 

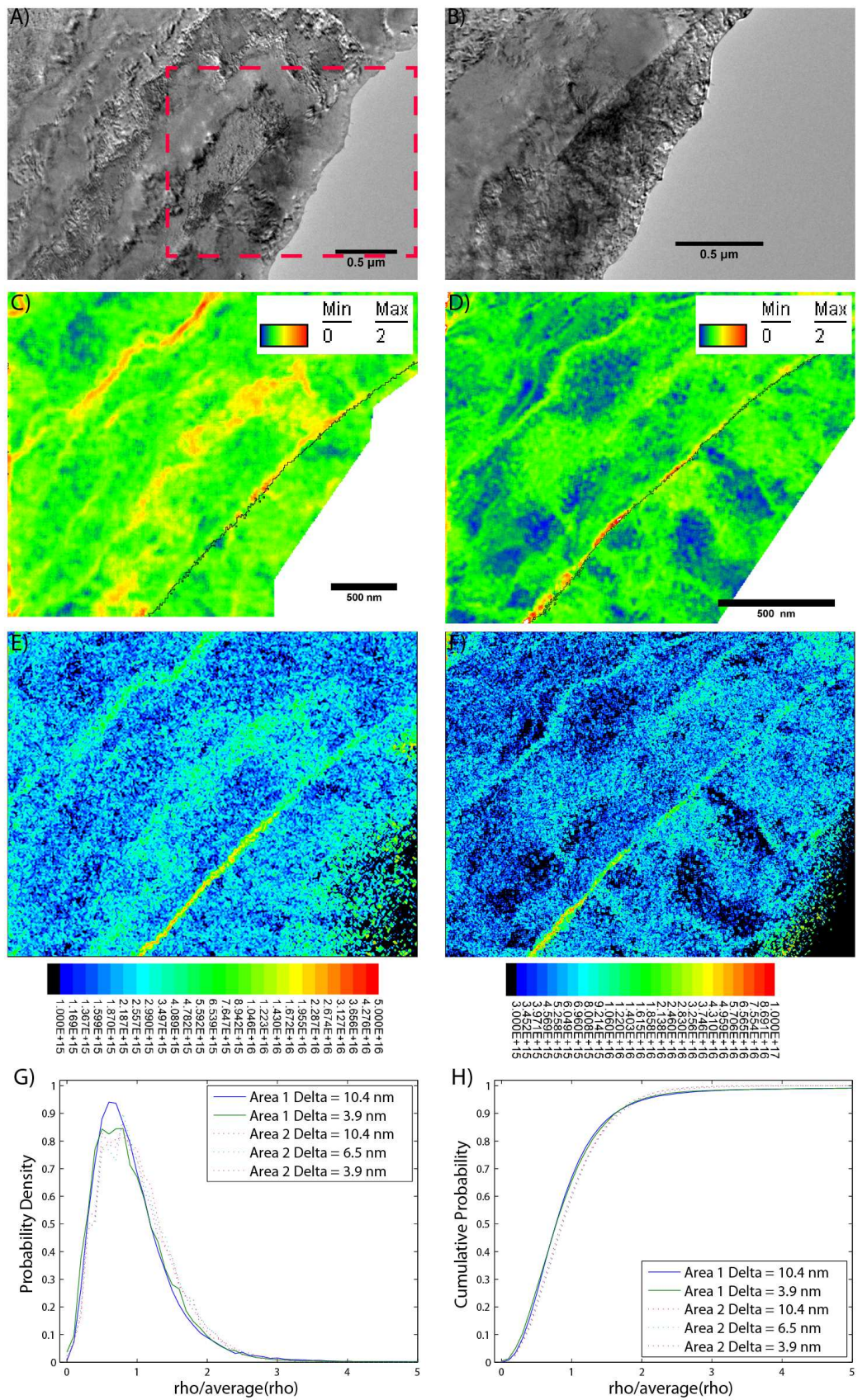
Figure 6. A\&B) TEM micrographs of the scan area, where B is at an increased magnification.
411 C\&D) Local orientation spread maps corresponding to the region shown in A \& B with step sizes 412 of 10.4 and $3.9 \mathrm{~nm}$, respectively. E\&F) Maps of estimated lower-bound GND density produced 413 from the same data as C \& D. G\&H) Normalized dislocation density probability distributions 414 and cumulative distributions, respectively. Area 1 is the region shown in A-F.

\section{4.4 Noise Effects and Image Enhancement}

416 Some amount of noise is present in the orientation data due to the angular resolution limit of the 417 indexing technique. This is of particular concern when the acquisition step size is varied since 
the minimum resolved dislocation density is inversely proportional to the step size [22]. In order to determine what role, if any, noise due to angular resolution plays in the high dislocation density values calculated from data acquired at very fine step sizes, three approaches were taken.

421 First, various amounts of noise were added to an orientation data set prior to Nye tensor analysis.

422 Each data point had a 50\% probability of being rotated a set amount - analogous to a minimum

423 angular resolution - about a random axis of rotation. As can be seen in Figure 7, increasing the

424 amount of noise in the orientation data causes an increase in calculated dislocation density.

425 Unlike the increased densities calculated at reduced step sizes, there is a significant change in the 426 cumulative probability function associated with this shift. Additionally, whereas with decreased 427 step sizes the dislocation structures become sharper and more clearly correspond with the 428 features visible in the micrograph, increasing the noise causes the features to be obscured as one 429 would expect. Furthermore, it takes an addition of $5^{\circ}$ random rotations to produce an increase in 430 dislocation density of approximately $1 \times 10^{16} \mathrm{~m}^{-2}$, approaching the scale of the densities calculated 431 at the smallest step sizes used.
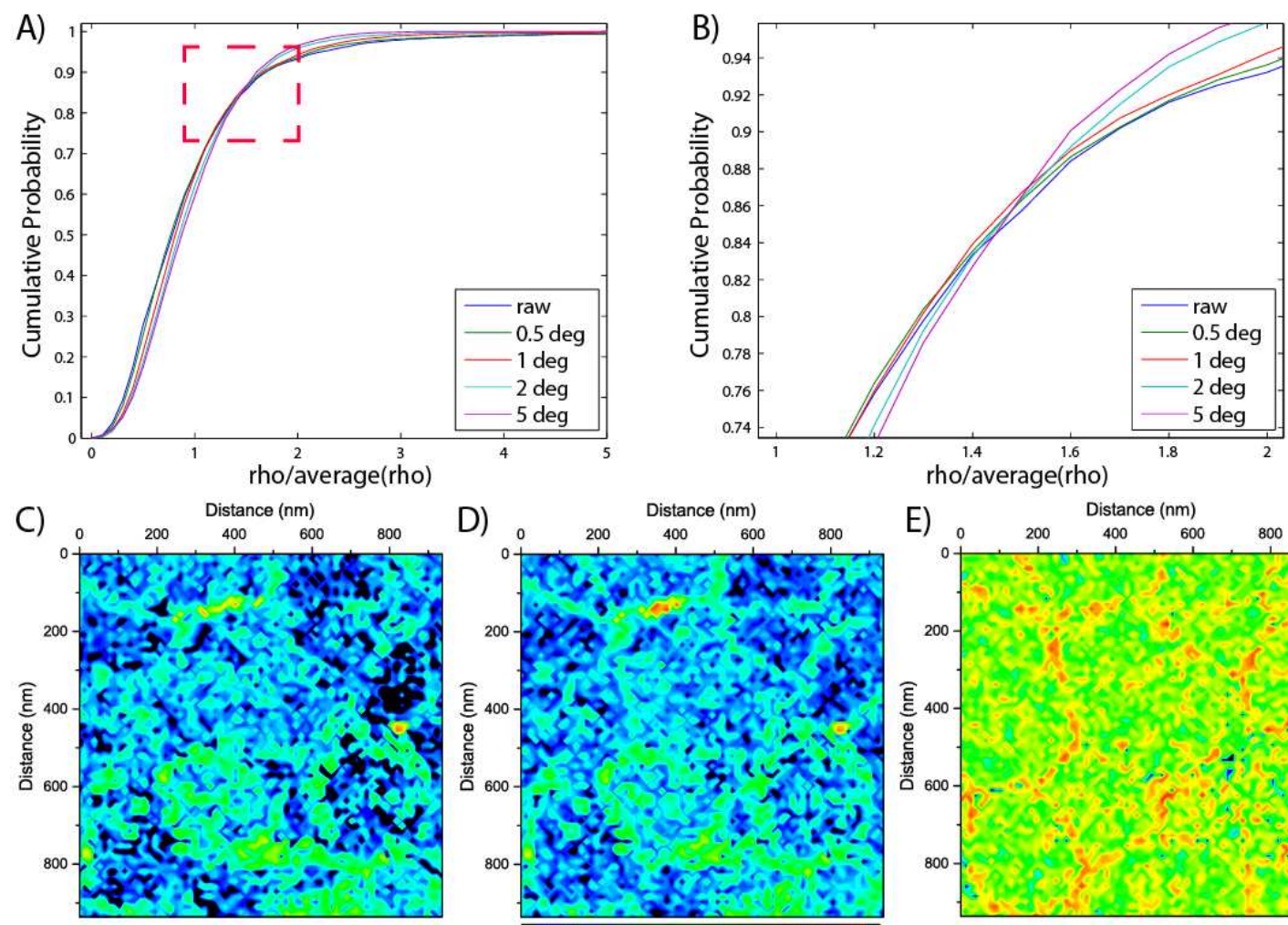

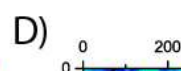

Distance (nm)

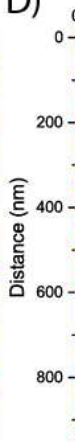

Figure 7. A) Cumulative probability distribution functions for the dislocation density data shown in C) with various levels of noise added. B) inset region from A showing the crossover at $\rho / \rho_{\text {avg }}=$ 1.5. The higher the level of noise, the less likely that a given point will have a density below this 

orientation data with a $5^{\circ}$ rotation added.

439 Second, a low-pass Gaussian discrete Fourier filter was applied to dislocation density data sets in 440 order to quantify the effects of noise on the values and to improve the image quality. As can be 441 seen in Figure 8C\&D, this filtering reduces the visual noise in the image by removing any high 442 frequency shifts in dislocation density such as those associated with the angular resolution limit. 443 Quantitatively, it produces a decrease in mean dislocation density on the order of $10^{11} \mathrm{~m}^{-2}$, even 444 for scans taken at reduced step sizes. This indicates that high frequency noise is not responsible 445 for the increase in calculated densities in this data relative to data acquired at larger step sizes.

446 Finally, two methods were used in an effort to enhance the effective angular resolution of the 447 technique. First, a Kuwahara filter was applied to the orientation data after indexing was 448 completed according to the method described by Humphreys, et al. [44]. A kernel size of five 449 pixels was used for filtering, with the quadrant with the lowest variance being assigned as the 450 new orientation for the center point in the kernel. This type of filtering minimizes small 451 fluctuations in the data such as those caused by high frequency noise while sharpening edge 452 features. It was chosen because recent work by Ghamarian, et al. suggests that this is an ideal 453 way to enhance the angular resolution of ACOM data. By comparing Figure 8E\&F with A\&B, 454 however, it is apparent that in this case Kuwahara filtering resulted in substantive changes to the 455 calculated dislocation structure. Moreover, due to the counterbalancing effects of smoothing 456 small fluctuations and emphasizing large ones, the filter does not cause a systematic decrease in 457 the average dislocation density and in some cases actually causes the average to increase.

458 The second method used to enhance the angular resolution of the ACOM data was an 459 interpolation algorithm described in detail recently by Rauch and Veron [39]. By interpolating 460 between the positions and intensities of spots in the neighboring template patterns that best fit the 461 experimental diffraction pattern, the orientation can be estimated with more precision 462 (approximately $0.3^{\circ}$ ) than the sampling of orientation space represented by the template bank. As 463 can be seen in Figure 8G\&H, the morphology of the calculated dislocation structures did not 464 change significantly when this refinement was utilized. Most importantly, this method does not 465 cause a systematic decrease in the average dislocation density. This indicates that the increases in 466 dislocation density seen at small step sizes is not due to angular resolution constraints because if 467 that were so then improving the angular resolution while holding the step size constant ought to 468 cause a corresponding decrease in density values. 

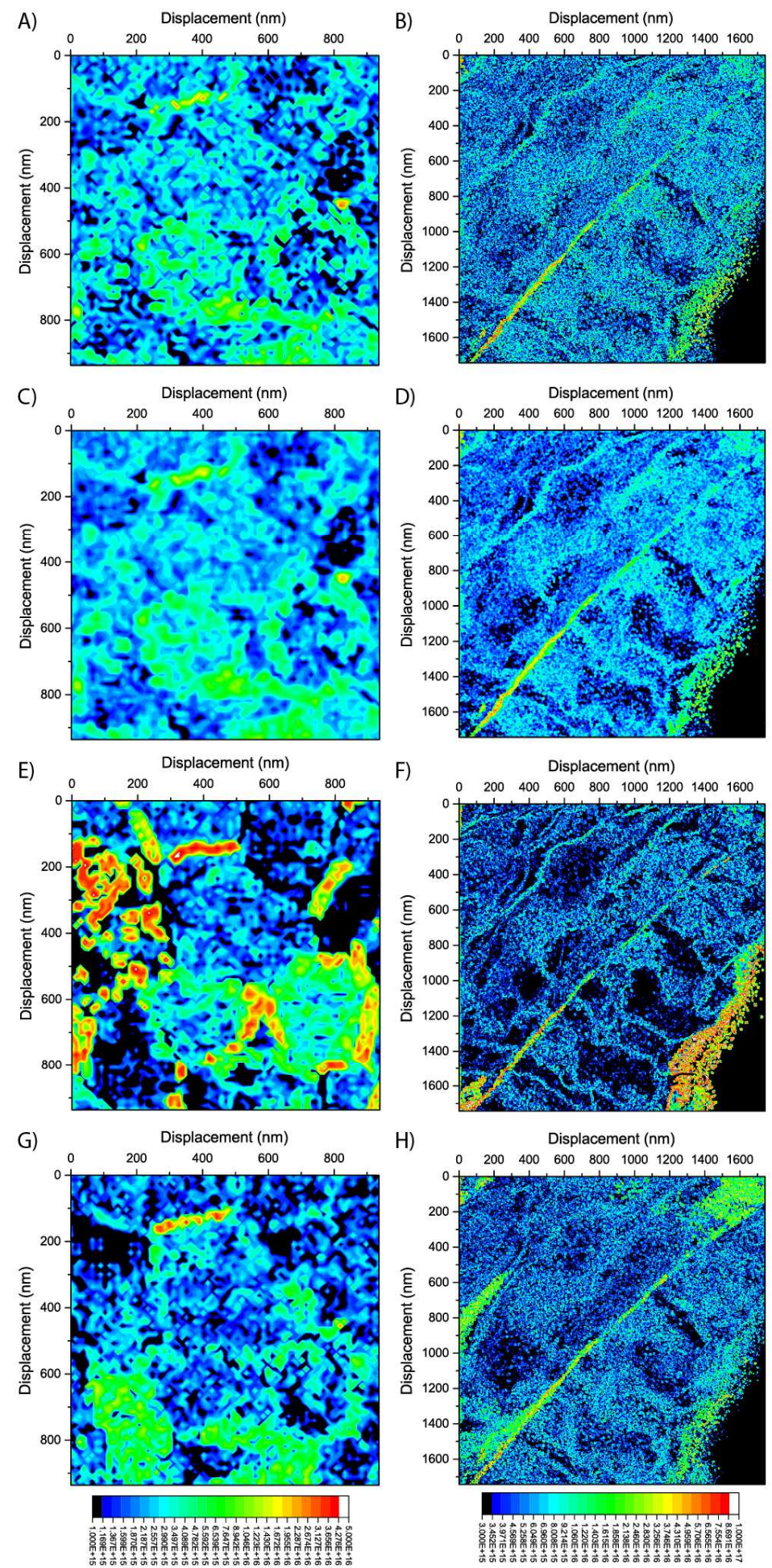

470 Figure 8. A\&B) GND density maps produced from raw data (no filtering). C\&D) GND density 471 maps for the same regions as A\&B produced from data that heen filtered using discrete 472 Fourier transform analysis. E\&F) GND density maps produced using Kuwahara filtering. G\&H) 473 GND density maps produced using interpolation method to refine the angular resolution. 


\section{4.5 Dislocation Motion and Dislocation-Driven Processes}

475 By applying this technique before and after an external stimulus during in situ processing, such 476 as the application of heat or strain, motion (e.g, annihilation or emission) of dislocations can be

477 quantified. One example of this, shown below, is the quantification of grain rotation through the 478 emission of dislocations from a triple junction during annealing. Figure 9 shows a region 479 adjacent to a triple junction in $\mathrm{Cu}$ before, during, and after annealing in situ at $720{ }^{\circ} \mathrm{C}$. As shown 480 in the video stills in Figure 9D, during the annealing process dislocations were emitted from the 481 triple junction which then glide approximately $800 \mathrm{~nm}$ into the matrix of the lower-right grain 482 before piling up. As a result of this process the corner of the grain is rotated, creating a sub-grain 483 with a disorientation of $3.5^{\circ}$ relative to the original matrix grain as shown in the misorientation 484 vs. displacement plot (Figure 9E). As can be seen in Figure 9C, the dislocation density map from 485 prior to this process indicates that the grain had a uniform dislocation distribution. The data 486 recorded after heating, on the other hand, clearly shows the build-up of dislocations where the 487 sub-boundary has formed (Figure 9G\&H). Grain rotation is just one example of a process of 488 microstructural evolution that can be informed by this technique. The mechanism and driving 489 force for twinning is of particular interest and is the subject of a parallel work using this 490 methodology [34]. 

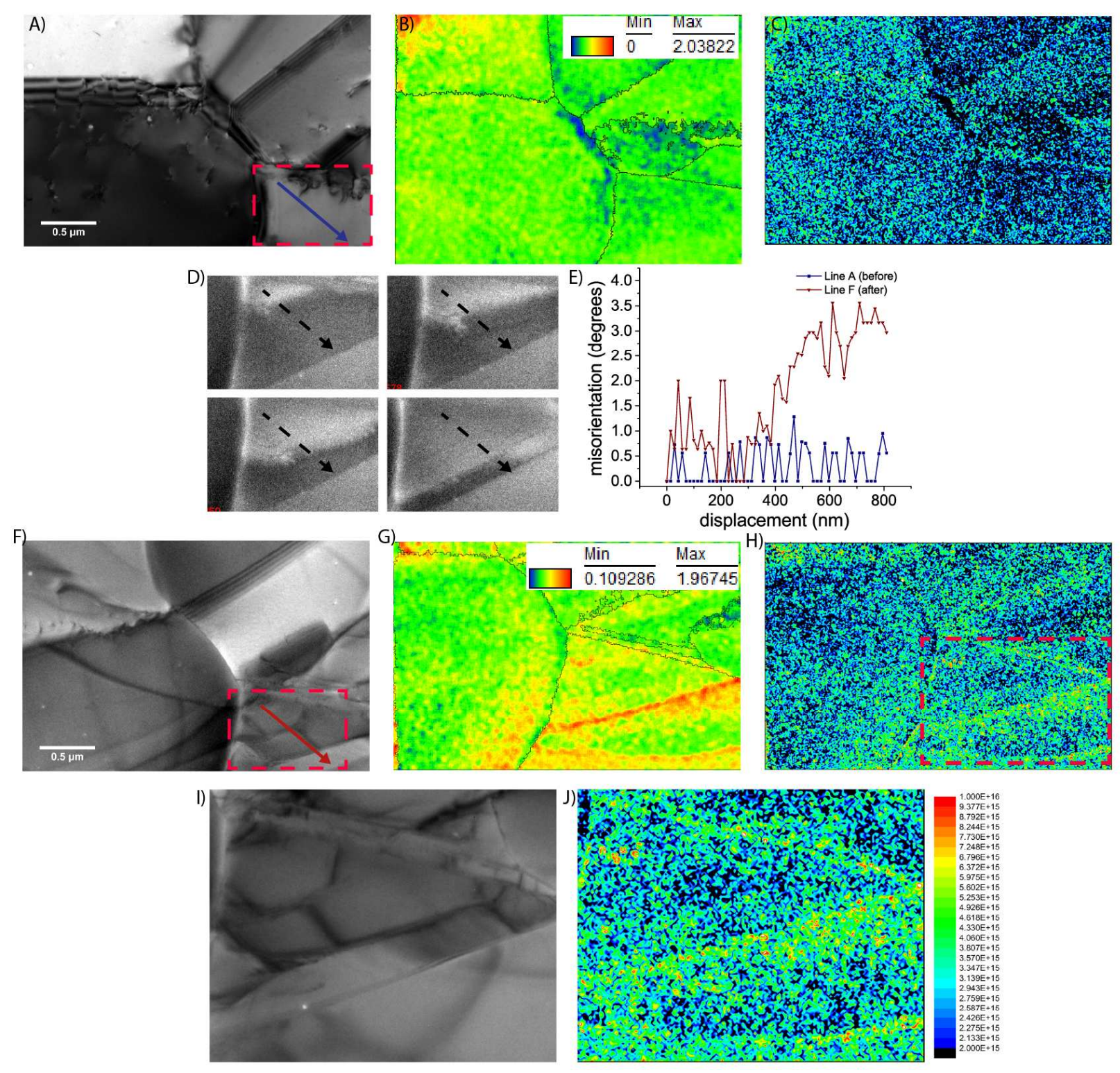

Figure 9. A\&F) TEM micrographs showing two triple junctions in a $\mathrm{Cu}$ foil before and after in situ annealing, respectively. B\&G) LOS maps corresponding to the images shown in A and F, respectively. $\mathrm{C} \& \mathrm{H}$ ) Dislocation density maps corresponding to the images shown in $\mathrm{A}$ and $\mathrm{F}$, respectively. D) Video stills from in situ heating showing dislocations being emitted and piling up in the region outlined in A and F. E) Plot of misorientation with respect to origin along the lines shown in A and F. I) TEM micrograph showing the area outlined in E at higher magnification. J) Region of GND map shown in H corresponding to the area shown in I.

\section{Conclusions}

502 misorientation analysis methods and Nye's dislocation theory to the data acquired by precession- 
using ACOM-TEM data have been demonstrated - local orientation spread mapping and Nye

The local orientation spread approach provides relative values of strain from point to point for a given region. It is a fast, convenient method for discerning relative dislocation density values from PED-ACOM data that can be carried out using commercially available software. The values produced are essentially a scalar estimation of local strain that can be compared directly from sample to sample for a given material and consistent acquisition parameters. This method is applicable to all known crystal structures as long as their diffraction patterns can be indexed accurately. The primary drawback of this method is that the contributions of elastic and plastic strain cannot be decoupled from one another so the individual strain tensor components cannot be determined.

The estimated lower-bound GND density approach provides an approximate measurement of the dislocation density at a given point. These values are directly comparable to one another because they are a measurement of the number of defects present. The method described herein is similar in approach to the technique described recently by Ghamarian, et al. [33] and produces calculated defect densities on the same orders of magnitude. The application of a hard angular limit for inclusion in calculations and least squares fitting of neighbor points in the present work instead of Kuwahara filtering leads to a very different final product. This method is easily adapted to BCC materials and other structures that contain only a single family of dislocations. It can be adapted to other crystal structures through the calculation of a solution to the Nye tensor that minimizes the line energy of the dislocations while accommodating the curvature [45] that describes all the dislocation types present and their relative frequency. Due to its quantitative nature, the analysis described herein is a powerful tool for measuring dislocation densities in TEM specimens that has the potential to inform many different processes of microstructural change, especially when combined with in situ TEM studies. Moreover, this method is not limited to PED-ACOM data; without modification it can be applied to EBSD data acquired in the SEM.

Due to the short length-scale of the measurements described herein, the dislocation densities reported ought to take into account some fraction of the SSDs present as well as the GNDs. Measurements of the same regions at multiple step sizes indicate that this is the case. A simplified sampling model was produced in order to approximate the effects of acquisition parameters on the resulting measurements. This model demonstrated the potential of this type of analysis to access the complete dislocation content of a specimen when a suitable combination of spot and step sizes are used in acquisition. A more detailed mathematical model must be developed in order to estimate more precisely what fraction of the total dislocation density is being measured using a given set of acquisition parameters.

Increased accuracy for both methods described herein can be achieved by the improvement of the indexing for the diffraction patterns in order to increase angular resolution. Likewise, 
542 improved spatial resolution can be achieved using a finer probe and step size. The effects of

543 elastic strain present in the material on the calculated dislocation densities must be determined in

544 order to ensure the accuracy of the calculated densities, although previous work has found their

545 contribution to be negligible for similar methods applied to EBSD. This could be achieved by

546 the analysis of diffraction spot positions relative to a reference or simulated pattern of the same

547 orientation, or by utilizing automated CBED, in which the strains are more readily accessible.

548 Coupling this method of quantitative dislocation analysis with orientation-based methods for

549 qualitatively resolving strain local strain yields a superior understanding of microstructural

550 events and thus holds key advantages for spatially resolving dislocation- dependent phenomena

551 in various materials.

\section{Acknowledgements}

554 Authors ACL and MLT gratefully acknowledge funding from the United States Department of 555 Energy Basic Energy Sciences (DOE/BES) under the Early Career program through contract

556 DE-SC0008274, funding from the National Science Foundation's Faculty Early Career Program 557 under contract \#1150807, and funding from the Department of Energy's Nuclear Energy

558 University Program under contract NE0000315.

\section{References}

560 [1] J. Burke, D. Turnbull, Recrystallization and grain growth, Prog. Met. Phys. (1952) 220292.

[3] F.J. Humphreys, M. Hatherly, Recrystallization and Related Annealing Phenomena, 2nd ed., Elsevier Inc., New York, NY, 1996.

[4] Y. Huang, F.J. Humphreys, Measurements of grain boundary mobility during recrystallization of a single-phase aluminium alloy, Acta Mater. 47 (1999) 2259-2268.

[5] A.D. Rollett, D.J. Srolovitz, R.D. Doherty, M.P. Anderson, Computer simulation of recrystallization in non-uniformly deformed metals, Acta Metall. 31 (1989) 627-639. using statistically stored dislocations in commercial purity aluminum, Metall. Mater. Trans. A. 37 (2006) 19-25. 
574

575

576

577

578

579

580

581

582

583

584

585

586

587

588

589

590

591

592

593

594

595

596

597

598

599

600

601

602

603

604

605

[7] R. Vincent, P. Midgley, Double conical beam-rocking system for measurement of integrated electron diffraction intensities, Ultramicroscopy. 53 (1994) 271-282.

[8] E.F. Rauch, M. Veron, Coupled microstructural observations and local texture measurements with an automated crystallographic orientation mapping tool attached to a tem, Materwiss. Werksttech. 36 (2005) 552-556.

[9] E.F. Rauch, J. Portillo, S. Nicolopoulos, D. Bultreys, S. Rouvimov, P. Moeck, Automated nanocrystal orientation and phase mapping in the transmission electron microscope on the basis of precession electron diffraction, Zeitschrift Für Krist. 225 (2010) 103-109.

[10] J. Portillo, E.F. Rauch, S. Nicolopoulos, M. Gemmi, D. Bultreys, Precession Electron Diffraction Assisted Orientation Mapping in the Transmission Electron Microscope, Mater. Sci. Forum. 644 (2010) 1-7.

[11] J.F. Nye, Some geometrical relations in dislocated crystals, Acta Metall. 1 (1953) 153162.

[12] a Arsenlis, D.. Parks, Crystallographic aspects of geometrically-necessary and statistically-stored dislocation density, Acta Mater. 47 (1999) 1597-1611.

[13] T.J. Ruggles, D.T. Fullwood, Estimations of bulk geometrically necessary dislocation density using high resolution EBSD., Ultramicroscopy. 133C (2013) 8-15.

[14] B.S. El-Dasher, B.L. Adams, A.D. Rollett, Viewpoint: experimental recovery of geometrically necessary dislocation density in polycrystals, Scr. Mater. 48 (2003) 141145 .

[15] S.I. Wright, B.L. Adams, Automatic Analysis of Electron Backscatter Diffraction Patterns, Met. Trans. A. 23A (1992) 759-767.

[16] J. Kysar, Y. Gan, T. Morse, X. Chen, M. Jones, High strain gradient plasticity associated with wedge indentation into face-centered cubic single crystals: Geometrically necessary dislocation densities, J. Mech. Phys. Solids. 55 (2007) 1554-1573.

[17] J.W. Kysar, Y. Saito, M.S. Oztop, D. Lee, W.T. Huh, Experimental lower bounds on geometrically necessary dislocation density, Int. J. Plast. 26 (2010) 1097-1123.

[18] W. Pantleon, Resolving the geometrically necessary dislocation content by conventional electron backscattering diffraction, Scr. Mater. 58 (2008) 994-997.

[19] A.J. Wilkinson, G. Meaden, D.J. Dingley, High-resolution elastic strain measurement from electron backscatter diffraction patterns: new levels of sensitivity., Ultramicroscopy. 106 (2006) 307-13. 
[20] A.J. Wilkinson, D. Randman, Determination of elastic strain fields and geometrically necessary dislocation distributions near nanoindents using electron back scatter diffraction, Philos. Mag. 90 (2010) 1159-1177.

[21] P.D. Littlewood, T.B. Britton, A.J. Wilkinson, Geometrically necessary dislocation density distributions in Ti-6Al-4V deformed in tension, Acta Mater. 59 (2011) 64896500 .

[22] J. Jiang, T.B. Britton, A.J. Wilkinson, Measurement of geometrically necessary dislocation density with high resolution electron backscatter diffraction: effects of detector binning and step size., Ultramicroscopy. 125 (2013) 1-9.

[23] T. Hardin, B.L. Adams, D.T. Fullwood, R.H. Wagoner, Estimation of the Full Nye Tensor by EBSD-Based Dislocation Microscopy, Mater. Sci. Forum. $702-703$ (2011) 489-492.

[24] T.J. Hardin, B.L. Adams, D.T. Fullwood, R.H. Wagoner, E.R. Homer, Estimation of the full Nye's tensor and its gradients by micro-mechanical stereo-inference using EBSD dislocation microscopy, Int. J. Plast. 50 (2013) 146-157.

[25] S.T. Wardle, L.S. Lin, A.D. Cetel, B.L. Adams, Orientation imaging microscopy: Monitoring residual stress profiles in single crystals using and imaging quality parameter, IQ, Proc. 52nd Annu. Meet. Microsc. Soc. Am. (1994) 680-681.

[26] S.I. Wright, M.M. Nowell, D.P. Field, A review of strain analysis using electron backscatter diffraction., Microsc. Microanal. 17 (2011) 316-29.

[27] D.P. Field, Quantification of partially recrystallized poly- crystals using electron backscatter diffraction, Mater. Sci. Eng. A. 190 (1995) 241-246.

[28] J. Wheeler, E. Mariani, S. Piazolo, D.J. Prior, P. Trimby, M.R. Drury, The weighted Burgers vector: a new quantity for constraining dislocation densities and types using electron backscatter diffraction on 2D sections through crystalline materials, J. Microsc. 233 (2009) 482-494.

[29] D. Chen, J.-C. Kuo, W.-T. Wu, Effect of microscopic parameters on EBSD spatial resolution., Ultramicroscopy. 111 (2011) 1488-94.

[30] P.W. Trimby, Orientation mapping of nanostructured materials using transmission Kikuchi diffraction in the scanning electron microscope., Ultramicroscopy. 120 (2012) 16-24.

[31] P. Moeck, S. Rouvimov, I. Häusler, W. Neumann, S. Nicolopoulos, Precession electron diffraction \& automated crystallite orientation / phase mapping in a transmission electron microscope, in: IEEE Int. Conf. Nanotechnol., 2011: pp. 754-759. 
[32] A.C. Leff, Evaluation of Strain Effects on Grain Boundary Engineering Mechanisms Using In Situ TEM, Drexel University, 2013.

[33] I. Ghamarian, Y. Liu, P. Samimi, P.C. Collins, Development and application of a novel precession electron diffraction technique to quantify and map deformation structures in highly deformed materials — as applied to ultrafine-grained titanium, Acta Mater. 79 (2014) 203-215.

[34] A.C. Leff, C.R. Weinberger, M.L. Taheri, Quantitative assessment of the driving force for twin formation utilizing Nye tensor dislocation density mapping, Acta Mater. to Be Submitt. (2014).

[35] P.D. Littlewood, a. J. Wilkinson, Geometrically necessary dislocation density distributions in cyclically deformed Ti-6Al-4V, Acta Mater. 60 (2012) 5516-5525.

[36] R. a. Renzetti, H.R.Z. Sandim, R.E. Bolmaro, P. a. Suzuki, a. Möslang, X-ray evaluation of dislocation density in ODS-Eurofer steel, Mater. Sci. Eng. A. 534 (2012) 142-146.

[37] M.W. Lai, D. Rubin, E. Krempl, Introduction to Continuum Mechanics, Third, Pergamon Press, Oxford, UK, 1993.

[38] D.P. Field, P.B. Trivedi, S.I. Wright, M. Kumar, Analysis of local orientation gradients in deformed single crystals., Ultramicroscopy. 103 (2005) 33-9.

[39] E.F. Rauch, M. Véron, Automated crystal orientation and phase mapping in TEM, Mater. Charact. 98 (2014) 1-9.

[40] J.-J. Fundenberger, a Morawiec, E. Bouzy, J.S. Lecomte, Polycrystal orientation maps from TEM., Ultramicroscopy. 96 (2003) 127-37.

[41] C. Feltner, C. Laird, Cyclic stress-strain response of FCC metals and alloys-II Dislocation structures and mechanisms, Acta Metall. 15 (1967).

[42] R. Wu, N. Pettersson, A. Martinsson, R. Sandström, Cell structure in cold worked and creep deformed phosphorus alloyed copper, Mater. Charact. 90 (2014) 21-30.

[43] J. Jiang, T.B. Britton, a. J. Wilkinson, Evolution of dislocation density distributions in copper during tensile deformation, Acta Mater. 61 (2013) 7227-7239.

[44] F. Humphreys, P. Bate, P. Hurley, Orientation averaging of electron backscattered diffraction data, J. Microsc. 201 (2001) 50-58.

[45] T. Ben Britton, S. Birosca, M. Preuss, A.J. Wilkinson, Electron backscatter diffraction study of dislocation content of a macrozone in hot-rolled Ti-6Al-4V alloy, Scr. Mater. 62 (2010) 639-642. 
671 Appendix A - One-dimensional model of orientation-based sampling for dislocation 672 measurement

673 In order to examine the effects of the data acquisition parameters on the measured dislocation 674 density using orientation-based sampling, a simple model was developed using MATLABß. The

675 sampling model begins by defining a one-dimensional specimen of length $L$ with dislocations 676 evenly spaced a distance of 1 arbitrary unit apart, starting at a user-defined offset from the edge

677 of the specimen. In this way, the dislocation density is set equal to 1. Each dislocation is assigned 678 either a positive or negative sign, which constitutes its Burgers vector. Two adjacent dislocations 679 with opposite signs constitute a statistically stored dislocation dipole, whereas consecutive 680 dislocations of the same sign are geometrically necessary. Various schemes for assigning signs to 681 the dislocations were tested such as all positive (all GNDs), alternating signs (all SSDs), every 682 third dislocation negative, and random assignment. Each dislocation causes an arbitrary rotation 683 of equal magnitude to the specimen with the direction of the rotation dependent on the sign.

684 Once the simulated structure has been generated, $N$ measurements of the average orientation of 685 the specimen across a probe diameter $D$ are taken at a distance $\Delta$ apart. $D$ is equivalent to the 686 beam spot size and $\Delta$ is equivalent to the step size for diffraction pattern acquisition. A

687 simplified contortion tensor $\mathrm{K}$ was then calculated as the difference in orientation between the 688 two nearest neighbors of each measurement divided by the distance between those two

689 measurements $(2 \Delta)$. The measured dislocation density for the specimen was calculated according 690 to:

$$
\rho_{\text {tot }}=\sum|\mathrm{K}| * \Delta / \mathrm{L}
$$

692 In addition, the fraction of the measured density that is attributable to GNDs was calculated 693 according to:

$$
\rho_{G N D}=\sum \mathrm{K} * \Delta / \mathrm{L}
$$

695 By comparing the values of $\rho_{\mathrm{tot}}$ and $\rho_{\mathrm{GND}}$ the combinations it was shown that dislocations that are 696 not geometrically necessary were resolved, especially at spot sizes lower than the dislocation 697 spacing. 